\title{
Article
}

\section{Performance on the traditional and the touch screen, tablet versions of the Corsi Block and the Tower of Hanoi tasks}

Robinson, Sarita Jane and Brewer, Gayle

Available at http://clok.uclan.ac.uk/14044/

Robinson, Sarita Jane ORCID: 0000-0002-4237-5412 and Brewer, Gayle ORCID: 0000-0003-0690-4548 (2016) Performance on the traditional and the touch screen, tablet versions of the Corsi Block and the Tower of Hanoi tasks. Computers in Human Behavior, 60 . pp. 29-34. ISSN 07475632

It is advisable to refer to the publisher's version if you intend to cite from the work. http://dx.doi.org/10.1016/j.chb.2016.02.047

For more information about UCLan's research in this area go to http://www.uclan.ac.uk/researchgroups/ and search for <name of research Group>.

For information about Research generally at UCLan please go to http://www.uclan.ac.uk/research/

All outputs in CLoK are protected by Intellectual Property Rights law, including Copyright law. Copyright, IPR and Moral Rights for the works on this site are retained by the individual authors and/or other copyright owners. Terms and conditions for use of this material are defined in the policies page. 
Performance on the traditional and the touch screen, tablet versions of the Corsi Block and the Tower of Hanoi tasks

Sarita J. Robinson* and Gayle Brewer

School of Psychology, University of Central Lancashire, Preston, PR1 2HE, UK

*Corresponding author. School of Psychology, University of Central Lancashire, Preston, Lancashire, PR1 2HE, UK. Email: SJRobinson1@UCLan.ac.uk 
- Traditional and tablet based Tower of Hanoi and Corsi Block tasks were compared

- Performance (i.e. number of moves and span length) did not differ between versions

- The computerized Tower of Hanoi task was completed more quickly

- Differences were identified for subjective workload and enjoyment

- Traditional and tablet versions of the cognitive tasks are largely equivalent

Keywords: Hanoi; Corsi; Cognitive Testing; Electronic; Computerized 
Psychologists routinely administer cognitive tasks to assess a range of mental abilities. In recent years, researchers and practitioners have employed new (i.e. digital) technologies to test cognitive performance, with tablet computer based tasks often replacing traditional versions. However, the extent to which findings from traditional and tablet based tasks are equivalent remains unclear. In the present study, sixty participants (18 men and 42 women) completed both the Tower of Hanoi and Corsi Block tasks in their traditional (wooden) form and using a touch screen tablet. Performance outcome measures (span length, number of moves and time taken) were recorded alongside subjective workload for each task. Findings revealed that number of moves and span length do not significantly differ between the traditional and tablet based versions of each task. However, the computerized Tower of Hanoi task was completed more quickly than the traditional version. Differences were noted for subjective workload with higher physical demand reported for the traditional versions of each task. Participants also reported the traditional Tower of Hanoi task to be more enjoyable but more mentally demanding. In conclusion, the touch screen versions of the Tower of Hanoi and Corsi Block tasks appear largely equivalent to the traditional versions. 


\section{Introduction}

Cognitive tasks are routinely administered by psychologists to assess a range of mental abilities. Two tasks that are extensively employed during psychological research studies are the Tower of Hanoi (credited to Édouard Lucas, 1883) and Corsi Block tasks (Corsi, 1972). The Tower of Hanoi task has been widely used with children, adolescents and adults (e.g. Guevara, Martinez, Aguirre, \& Gonzalez, 2012) from both general (Welsh \& Huizinga, 2005) and clinical (Gimenez, et al. 2003; Vakil, Hassin-Baer, \& Karni, 2014) samples. Similarly the Corsi Block task has been employed with children, adolescents and adults (e.g. Carvalho et al., 2014; Piccardi, et al., 2008) in both normative (Shah, Prados, Gamble, De Lillo, \& Gibson, 2013) and clinical (Stoffers, Berendse, Deijen, \& Wolters, 2003) populations. In recent years, researchers and practitioners have employed new (i.e. digital) technologies in order to test cognitive performance, often replacing traditional equipment based testing (Groves, 2011). Therefore, it is important to consider whether the new electronic based testing is equivalent to the traditional method of cognitive task administration.

Previous research has suggested that complex processes, such as reading medical information, can be completed equally well regardless of whether the information is presented on paper or on a computer screen. However, interestingly users' subjective ratings suggest that they preferred the paper format (Holzinger, Searle, Wernbacher, 2011). This indicated that even when information displayed digitally is processed as effectively as information on a computer screen, non-computerized versions can still be preferred. With regard to delivering cognitive testing in a computerized form research has suggested that using digital versions can have some advantages over traditional paper and pencil or apparatus based testing. For example, Chua (2012) found that participants took a shorter time to complete the tests and had higher levels of motivation when completing computer based 
compared to pen and paper based tasks. However, digital testing differs from noncomputerized testing in a number of ways. First, participant responses to cognitive tasks will be presented on a flat computer screen and so will display a 2D representation of the task.rather than the traditional wooden 3D apparatus form. Comparisons between traditional and computer based cognitive testing have revealed that computer based tasks may place greater cognitive demands on participants, with participants often reporting a higher workload for tasks presented on screen (Hart \& Staveland, 1998; Noyes, Garland, \& Robbins, 2004). As both the Tower of Hanoi and the Corsi Block tasks are thought to involve processing in the frontal lobes and be specially related to working memory functioning (Goela, Pullara, \& Grafman, 2001; Vandierendonck, Kemps, Fastame \& Szmalec, 2004; Welsh, Satterlee-Cartmell, \& Stine, 1999), increases in cognitive load as a result of presentation mode could impact on task performance. ) or

The second difference between traditional and digital cognitive tasks is the manner in which the participants makes a response. When using 3D apparatus the participant will receive a hapic experience has they can feel and handle the apparatus. The hapic experience is missing from when participants use indirect input device (such as a mouse or a keyboard). Research exploring presentation mode has found that the computerized Tower of Hanoi task using indirect input yielded more successful completion but that participants required more moves to achieve this. This may suggest that different strategies are being employed when the task is presented in a traditional or computerized form (Noyes \& Garland, 2003). Research investigating computer mediated presentation of the Corsi Block task has identified no difference in performance when completing traditional or computerized tasks using indirect input devices (Nelson, Dickson, \& Baños, 2000). However, these studies considered computerized tests which used indirect input devices such a mouse. Relatively little is known 
about the impact of using tablet based technologies, where a direct (i.e. touch screen) surface is used.

Touch screen technology has become increasing prevalent in recent years (Atkinson, 2008; Zickuhr, 2013). The use of touch screen cognitive testing has therefore become increasing possible. However, important physical, (Straker, et al., 2008) and behavioral (Chung, 2015) differences have been observed when people use direct (touch screen) compared to indirect (mouse or keyboard) input devices which may impact on or task performance Generally, touch screen technologies, which use finger touch on a sensitive screen, are thought to require less hand-eye coordination than input via a mouse or keyboard (Shneiderman, 1991). Further, touch screen technologies appear to offer a more immersive experience as the user can directly interact with the objects on the screen. This means that touch screen technologies are intuitive and so the user does not need to be computer literary in order to use them (Holzinger, 2003; Siegenthaler, Bochud, Wurtz, Schmid \& Bergamin, 2012). Therefore, the impaired cognitive load previously reported as a result of computer use may not be evident in when using touch screens for responses.. Indeed, it has been observed that patients with mild cognitive dysfunction found touch screen technology easier to use than other computer input devices (Deguchi, Kono, Deguchi, Morimoto, Kurata, et al. 2013).

Furthermore, touch screen responses may influence other factors known to affect working memory. Changing indirect input devices for touch screen technologies has been shown to impact on the way in which people interact with digital devices (Toy, Peres, David, Nery \& Phillips (2012) with people who use touchscreen devices feeling higher levels of engagement with computer based tasks such as online shopping (Chung, 2015). In addition, if factors such as fatigue (van der Linden, Frese, \& Meijman, 2003; Wästlund, Reinikka, 
Norlander, \& Archer, 2005) or mood (Phillips, Bull, Adams \& Fraser, 2002) are impacted by touch screen use then this may impact on the cognitive resources available to complete a cognitive task.

) .

In summary, previous research has considered the extent to which mode of presentation (i.e. traditional versus indirect input computer) can affect cognitive task performance. However, in recent years, tablet use (using direct input touch screens) has become particularly widespread and so the current study compares participant performance and perceptions of traditional wooden and touch screen based cognitive task presentation. Specifically, the current study compared electronic and wooden versions of the Tower of Hanoi and Corsi Block tasks. In the current study all participants were naive to the cognitive tasks employed as previous research has shown that cognitive task experience can lead to enhanced performance (Williams \& Noyes, 2007). Furthermore, other factors which may influence performance such a handedness, gender and competence with tablet computers were considered.

\section{Method}

\subsection{Design}

A 2 (Task: Tower of Hanoi, Corsi Block) X 2 (Form: traditional, tablet) repeated measures design was implemented. The order of presentation was counterbalanced using a Latin square design.

\subsection{Participants}

Men $(N=18)$ and women $(N=42)$ aged $18-49$ yrs $(M=25.93, S D=7.68)$ were opportunity sampled from the University of Central Lancashire. All participants had similar educational backgrounds (educated to first year degree level), reported high levels of 
familiarity with tablet technology and were naive to the Tower of Hanoi and Corsi Block tasks. Fifteen participants self-identified as left-handed.

\subsection{Materials}

Demographic questionnaire: Participants were asked to state their age, gender, handedness and prior experience using tablet computers.

NASA-TLX Perceived Workload Questionnaire (Hart \& Staveland, 1988): The NASA-TLX measures perceived workload on six visual analogue scales (mental demand, physical demand, temporal demand, effort, performance and frustration). The scale was amended to include a seventh visual analogue scale for enjoyment. Participants place a mark along the $10 \mathrm{~cm}$ line to express their perceived level of workload. The length (in millimeters) from the start of the line to the cross is measured with a minimum score of 0 to a maximum score of 100. A higher score indicates a higher perceived level of workload or enjoyment.

Tower of Hanoi Task: The five disk version of the Tower of Hanoi task consists of three pegs arranged in a line and five disks of decreasing sizes. At the start of the task, all disks are stacked on one peg, with the largest disk at the bottom, and the remaining disks stacked in decreasing size. The goal of the task is to move all disks to the end peg via the central peg following a set of rules. The rules state that (a) you can only move one disk at a time (b) a larger disk cannot be placed on a smaller disk and (c) all disks must remain on a peg unless they are being moved. The experimenter records the number of moves made and the time taken to complete the task. Noyes and Garland (2003) note that the puzzle can be completed in a relatively short amount of time with only 31 moves needed to solve the puzzle. Two versions of the Tower of Hanoi task were used in the present study. The first was a traditional wooden version and the second was a tablet version displayed on an iPad. In the iPad version participants selected the disk they wished to move by touching the screen where it was located. They then dragged the disk with their finger along the screen to the 
location of the peg they intended to move the disk to. The electronic version is produced by Hong (2011) and was downloaded from the iTunes store. Consistent with previous studies using the Tower of Hanoi task (e.g. Noyes \& Garland, 2003), no assistance was provided to participants who experienced difficulties with the task.

Corsi Block Task (Corsi, 1972): The apparatus for the traditional wooden version of the Corsi Block task consisted of a series of nine blocks (3 $\mathrm{cm}$ cubed) arranged irregularly on a $23 \times 28 \mathrm{~cm}$ board. The blocks were tapped by the experimenter in a pre-set randomly generated order at a rate of one block per second. The participant was then asked to repeat the sequence immediately after the experimenter had finished. The experimenter started with a two-block sequence and increased this by one block if participants correctly remembered the sequence in one or more of the two trials undertaken. If the participant got two trials incorrect for one sequence, the task was terminated. The block span was calculated as the longest sequence where the participant recalled at least one trial sequence correctly. The electronic version of the Corsi Block task was displayed on an iPad. The on screen 'finger' pointed to the routine which was to be followed. Participants then touched the screen with their finger. The iPad version of the task was created by Darby (2011) and downloaded from the iTunes store.

\subsection{Procedure}

Participants completed an initial demographic questionnaire. Participants then completed both electronic and wooden versions of the Tower of Hanoi and Corsi Block tasks. The order of task delivery was randomised using a Latin square design. Participants completed a NASA-TLX questionnaire after each task to monitor the perceived difficulty (i.e. workload) and enjoyment of the tasks and overall performance. No financial incentives were provided for participation.

\section{Results}


Factors previously found to impact on Tower of Hanoi and Corsi Block task performance were first considered. Comparisons (independent samples t tests) between left and right handed participants revealed that right-handed participants completed the electronic Tower of Hanoi task more quickly $(t(58)=2.04, p<.05, \mathrm{~d}=0.54)$ and in less moves $(t(58)=$ $2.62, p<.05, \mathrm{~d}=0.69)$ than left-handed participants. No other significant differences were observed. See Table 1 for the descriptive statistics (means and standard deviations). A series of independent t-tests exploring the effects of gender, tablet confidence (median split of high and low) found no effects on any of the tasks examined $(p<.05)$.

Table 1: Mean (SD) Cognitive Task Performance For Left and Right Handed Participants

\begin{tabular}{llll}
\hline Task & Outcome & Left Handed & Right Handed \\
\hline Traditional Corsi Block & Span Length & $6.00(1.06)$ & $6.04(.95)$ \\
& Time Taken (min) & $2.00(.78)$ & $2.17(.73)$ \\
Electronic Corsi Block & Span Length & $6.40(1.18)$ & $5.78(1.06)$ \\
& Time Taken (min) & $2.53(.95)$ & $2.01(.85)$ \\
Traditional Tower of Hanoi & Number of Moves & $4.52(2.44)$ & $3.46(2.03)$ \\
& Time Taken (min) & $75.73(39.56)$ & $58.16(23.16)$ \\
& Number of Moves & $3.47(2.14)$ & $2.41(1.62)$ \\
Electronic Tower of Hanoi & Time Taken (min) & $80.20(38.94)$ & $57.42(25.32)$
\end{tabular}

Participant performance on the traditional wooden and electronic versions of the Tower of Hanoi tasks and participant's perceptions of the two tasks was considered. Correlational analysis suggests that span length on Corsi block task was highly correlated between the electronic and wooden version $(\mathrm{r}=.39, \mathrm{p}<.01)$. Performance (number of moves) 
was highly correlated on both versions of the Tower of Hanoi task $(r=.37, p<.01)$.A series of independent t-tests was undertaken to compare performance order (whether participants undertook the electronic or wooden version of the two tasks first. No differences were found in performance or time taken on either the Tower of Hanoi or the Corsi block tasks $(p<.05)$. Table 2 shows the descriptive statistics (means and standard deviations) for the two task presentations.

Table 2: Mean (SD) Tower of Hanoi Task Performance and Participant Perceptions (Traditional and Electronic Presentation)

\begin{tabular}{lll}
\hline Outcome & Traditional & Electronic \\
\hline Time Taken (min) & $3.73(2.17)$ & $2.67(1.81)$ \\
Number of Moves & $62.55(28.82)$ & $63.12(30.61)$ \\
Mental Demand & $72.05(22.78)$ & $61.78(28.19)$ \\
Physical Demand & $35.62(30.04)$ & $21.62(26.37)$ \\
Temporal Demand & $54.37(26.88)$ & $52.18(30.87)$ \\
Effort & $68.37(19.76)$ & $62.02(27.06)$ \\
Performance & $58.72(24.71)$ & $60.90(25.97)$ \\
Frustration & $50.10(32.32)$ & $45.00(32.52)$ \\
Enjoyment & $75.28(21.17)$ & $68.72(23.82)$
\end{tabular}

Paired samples t-tests showed no difference in performance (number of moves needed to complete the task) between the traditional and tablet versions of the task $(t(59)=-.13, p=$ .90). The time taken to complete the electronic version of the task was however significantly shorter than the traditional version $(t(59)=3.56, p=.01, \mathrm{~d}=0.93)$. Participants found the traditional wooden version of the Tower of Hanoi task to be more mentally $(t(59)=2.59, p=$ 
$.01, \mathrm{~d}=0.67)$ and physically demanding $(t(59)=4.37, p<.01, \mathrm{~d}=1.14)$ and more enjoyable $(t(59)=2.21, p=.03, \mathrm{~d}=.58)$. There was no difference in the extent to which participants found the tasks temporally demanding $(t(59)=.56, p=.58)$, frustrating $(t(59)=1.29, p=.20)$, effortful $(t(59)=1.88, p=.07)$ or their perceived performance $(t(59)=-.57, p=.57)$.

Participant performance on the traditional wooden and electronic versions of the Corsi Block tasks and participant's perceptions of the two tasks was considered. Table 3 shows the descriptive statistics (means and standard deviations) for the two task presentations.

Table 3: Mean (SD) Corsi Block Task Performance and Participant Perceptions (Traditional and Electronic Presentation)

\begin{tabular}{lll}
\hline Outcome & Traditional & Electronic \\
\hline Time Taken (min) & $2.13(.74)$ & $2.14(.90)$ \\
Number of Moves & $6.03(.97)$ & $5.93(1.12)$ \\
Mental Demand & $73.68(17.58)$ & $69.92(18.84)$ \\
Physical Demand & $28.58(29.09)$ & $20.10(22.75)$ \\
Temporal Demand & $44.53(28.55)$ & $48.32(25.21)$ \\
Effort & $66.42(21.74)$ & $65.90(21.30)$ \\
Performance & $52.48(21.98)$ & $57.12(21.39)$ \\
Frustration & $43.53(28.66)$ & $43.93(28.06)$ \\
Enjoyment & $61.13(25.36)$ & $64.40(26.15)$
\end{tabular}

Paired samples t-tests indicated no substantial difference in the time taken to complete the Corsi Block task $(t(59)=-.10, p=.92)$ or span length $(t(59)=.67, p=.51)$. Participants found the wooden version of the task to be more physically demanding $(t(59)=2.67, p=.01$, $\mathrm{d}=0.70)$. Perceptions of the extent to which the tasks were mentally $(t(59)=1.52, p=.13)$, or 
temporally demanding $(t(59)=-1.42, p=.16)$, and effortful $(t(59)=.23, p=.52)$ did not differ. Perceived performance $(t(59)=-1.43, p=.16)$, frustration $(t(59)=-1.11, p=.91)$ and enjoyment $(t(59)=-1.17, p=.25)$ were also similar.

\section{Discussion}

The present study investigated the equivalence of traditional and tablet based versions of two cognitive tasks. The results demonstrate that tablet and traditional versions of the Tower of Hanoi and Corsi Block tasks do not differ on key outcome measures (i.e. number of moves or span length). Participant also performed equally as well on both versions of each task with no observed differences in span length or moves taken between the tablet and traditional task version. . Participants were however significantly faster when completing the electronic compared to the traditional version of the Tower of Hanoi task. This finding suggests that researchers and practitioners should focus on number of moves as an outcome for this task rather than time taken. Interestingly, some differences were observed in the subjective workload reported by participants. The traditional tasks were rated as more physically demanding than their tablet based counterparts. In addition, the traditional version of the Tower of Hanoi task was rated as being more enjoyable as well as being more mentally demanding. Finally, two factors previously found to impact on task performance, gender and experience using tablet computers did not appear to influence task performance. However, handedness did effect performance on the Tower of Hanoi task when delivered in tablet form, with right-handed individuals performing the task more quickly and in less moves than left handed participants.

An important difference between the current study and previous research investigating computer presentation is the input device used. Previous studies reporting higher levels of workload for tasks presented on screen have used indirect input devices such as a computer mouse (Hart \& Staveland, 1988; Noyes, et al., 2004). It is possible that in those studies the 
use of an indirect input device was associated with an increased cognitive load i.e. participants employed some of the limited working memory resources available to use the input device and hence these resources were not directed at the cognitive task. In contrast, touch screens appear to be easier to use and more intuitive than indirect input devices (Holzinger, 2003; Siegenthaler et al., 2012). Hence, there may be no additional cognitive load demands placed on participants when using the tablet versions of the Tower of Hanoi and Corsi Block tasks.

Other factors which may affect working memory function and so may impact on working memory dependent cognitive task performance were examined. Self-reported subjective workload results gave no indication that tablet versions of the tasks increased fatigue (van der Linden, Frese, \& Meijman, 2003; Wästlund et al., 2005), with higher levels of physical demand attributed to the traditional wooden versions of both the Corsi Block and Tower of Hanoi tasks. Interestingly, the traditional wooden version of the Tower of Hanoi task was also associated with more enjoyment. Though mood has previously been associated with impairments in cognitive performance (Phillips et al., 2002) none were apparent in this study. Increased enjoyment by participants using the traditional apparatus could however lead to better participant engagement and continued participation. Additional work investigating the impact of mood on performance is recommended.

Another interesting finding was that left-handed people performed worse than righthanded participants on the electronic version of the Tower of Hanoi task. Holzinger, Scherer and Ziefle (2011) suggest that as the majority of computer users are right-handed, then designers may layout items on the screen in a way which is more intuitive for right-handers. This may then place left-handed people at a disadvantage when completing cognitive tasks. Alternatively, poorer performance on the Tower of Hanoi task in left-handed participants may reflect the higher levels of state anxiety displayed by left-handed people in novel 
situations (Wright, \& Hardie, 2012) and the disruption of visuospatial working memory caused by anxiety (Shackman et al., 2006).Future research may further compare the performance of left and right-handed participants on novel tablet based tasks.

Cognitive tests are typically administered in an experimental or clinical offline environment. The present findings suggest that cognitive tasks could also be administered via tablet computers either online or offline. Administration of cognitive tasks via tablet technologies may offer the researcher a number of practical advantages. For example, tablet based delivery may facilitate access to a wider and more ecologically valid sample (Musch \& Reips, 2000). Indeed, in recent years, there has been an increase in the use of online surveys to target populations that are difficult to access (Andrews, Nonnecke, \& Preece, 2003) or have shared motivations or interests (Wright, 2002). This form of data collection is time and cost effective and appears to produce data that is comparable to or of higher quality (e.g. fewer missing values) than offline studies (Llieva, Baron, \& Healey, 2002; Stanton, 2006; Yun \& Trumbo, 2000). In part, this may reflect the use of automatic scoring and coding systems, which can reduce experimenter error.

\section{Limitations}

The current study was reliant on a Western undergraduate sample reporting high levels of familiarity with tablet based technologies. Future studies should therefore seek to replicate these results with a more diverse sample. In particular, regional and cultural differences occur with regards to tablet focused anxiety (Pruet, Ang, \& Farzin, 2015) and attitudes towards tablets and desktop computers (Sung \& Mayer, 2012), which may each influence task performance or engagement. Caution should also be exercised when extrapolating findings to older populations. Older adults can have age-related cognitive and physical difficulties which can impact on their ability to use touch screen technology (Caprani, O’Connor, \& Gurrin, 2012). However, Holzinger, Searle \& Wernbacker (2001) 
suggest that an important factor to consider is an individual's previous exposure to technology, known as the PET factor. Studies have shown that older adults are less likely to use technology than the younger generation (Caprani,et al. 2012) but short periods of practice can result in proficiency (Kobayashi, Hiyama, Miura, Asakawa, Hirose et al. (2011). Therefore future research should investigate administration of cognitive tasks in tablet form to a more elderly population who maybe more unfamiliar with this technology and look at the impact of training.

This form of presentation may have a number of practical advantages, for example, more accessible delivery to people with motor impairments (perhaps as a result of a stroke, Deguchi et al. 2013). Indeed tablet based technologies may actually be more user friendly to an older populations as they require less motor dexterity and are less physically demanding than the traditional wooden versions. Though the use of tablet based technologies with older populations undergoing neurocognitive testing has been examined (Makizako, et al., 2012), further research is needed to ensure that tablet based testing is adapted for the physical needs of an older populations (Vasconcelos, Silva, Caseiro, Nunes, \& Teixeira, 2012).

The present study employed two cognitive tasks i.e. the Tower of Hanoi and Corsi Block tasks and findings have important implications for the use of these tasks which are widely administered to a range of populations (e.g. Carvalho, et al., 2014; Guevara et al., 2012). Results may not however be generalizable to other cognitive tasks. Future research may for example consider compare physical and computerised versions of variations of these tasks such as the Cyclic Towers of Antwerpen (Minsker, 2014) and Tower of London (Shallice, 1982) problems. Similarly, researchers may investigate the extent to which mode of presentation influences tasks assessing a wider range of cognitive abilities (e.g. memory, decision making). In future it may be interesting to look at the latent-level equivalence of the 
tasks and look to compare participant's tasks performance with a standardized measure of STM or WM.

To conclude, the current study investigated participant experiences of traditional and tablet based versions of the Tower of Hanoi and Corsi Block tasks. Mode of presentation did not impact on performance for either the Tower of Hanoi or Corsi Block task, though performance on the traditional version of the Tower of Hanoi task was significantly faster than the electronic version. Hence, tablet based cognitive tasks present a viable option to researchers seeking a practical alternative to traditional offline presentation. Indeed, the use of tablet based measures has important advantages compared to traditional formats (e.g. sustainability, data storage and coding, access to participants) and thus may be preferable.

\section{References}

Andrews, D., Nonnecke, B., \& Preece, J. (2003). Electronic survey methodology: A case study in reaching hard to involve Internet users. International Journal of HumanComputer Interaction, 16, 185-210. doi: 10.1207/S15327590IJHC1602_04

Atkinson, P. (2008). A bitter pill to swallow: The rise and fall of the tablet computer. Design Issues, 24, 3-25.

Caprani, N., O'Connor, N. E., \& Gurrin, C. (2012). Touch screens for the older user (pp. 95118). InTech.

Carvalho, C.F., Menezes-Filo, J.A., de Matos, V.P., Bessa, J.R., Coelho-Santos, J., Viana, G.F.S., Argollo, N., \& Abreu, N. (2014). Elevated airbourne manganese and low executive function in school-aged children in Brazil. NeuroToxicology, 45, 301-308.

Corsi, P.M. (1972). Human memory and medial temporal region of the brain. Dissertation Abstracts International, 34, 891B.

Chua, Y. P. (2012). Effects of computer-based testing on test performance and testing motivation. Computers in Human Behavior, 28(5), 1580-1586. 
Chung, S. (2015). Do Touch Screen Users Feel More Engaged? The Impact of Touch Interfaces on Online Shopping. Advances in Consumer Research, 43.

Darby, D. (2011). SpanTests. https://itunes.apple.com/gd/app/spantests/id479165359?mt=8

Deguchi, K., Kono, S., Deguchi, S., Morimoto, N., Kurata, T., Ikeda, Y., \& Abe, K. (2013). A Novel Useful Tool of Computerized Touch Panel-Type Screening Test for Evaluating Cognitive Function of Chronic Ischemic Stroke Patients. Journal of Stroke and Cerebrovascular Diseases, 22(7), e197-e206.

Gimenez, M., Junque, C., Perez, M., Vendrell, P., Baeza, I., Salamero, M., Mercader, J.M., \& Bernardo, M. (2003). Basal ganglia N-acetylaspartate correlates with the performance in the procedural task 'Tower of Hanoi' of neuroleptic-naïve schizophrenic patients. Neuroscience Letters, 347, 97-100. doi: 10.1016/S0304-3940(03)00698-0

Goela, V., Pullara, S.D., \& Grafman, J. (2001). A computational model of frontal lobe dysfunction: Working memory and the Tower of Hanoi task. Cognitive Science, 25, 287-313. doi: 10.1016/S0364-0213(01)00037-4

Groves, R.M. (2011). Three eras of survey research. Public Opinion Quarterly, 75, 861-871. doi: $10.1093 /$ poq/nfr057

Guevara, M.A., Martinez, L.E.R., Aguirre, F.A.R., \& Gonzalez, M.H. (2012). Prefrontalparietal correlation during performance of the towers of Hanoi task in male children, adolescents and young adults. Developmental Cognitive Neuroscience, 2, 129-138. doi: 10.1016/j.den.2011.05.002

Hart, S.G., \& Staveland, L.E. (1988). Development of NASA-TLX (Task Load Index): Results of empirical and theoretical research. In P.A. Hancock \& N. Meshkati (Eds). Human Mental Workload (pp 139-183). Amsterdam: Elsevier. 
Holzinger, A. (2003). Finger instead of mouse: touch screens as a means of enhancing universal access. In Universal access theoretical perspectives, practice, and experience (pp. 387-397). Springer Berlin Heidelberg.

Holzinger, A., Baernthaler, M., Pammer, W., Katz, H., Bjelic-Radisic, V., \& Ziefle, M. (2011). Investigating paper vs. screen in real-life hospital workflows: Performance contradicts perceived superiority of paper in the user experience. International Journal of Human-Computer Studies, 69(9), 563-570.

Holzinger, A., Scherer, R., \& Ziefle, M. (2011). Navigational user interface elements on the left side: intuition of designers or experimental evidence?. In Human-Computer Interaction-INTERACT 2011 (pp. 162-177). Springer Berlin Heidelberg.

Holzinger, A., Searle, G., \& Wernbacher, M. (2011). The effect of previous exposure to technology on acceptance and its importance in usability and accessibility engineering. Universal Access in the Information Society, 10(3), 245-260.

Hong, J. (2011). Tower of Hanoi -Pro. Retrieved from https://itunes.apple.com/us/app/towerof-hanoi-pro/id432096485?mt=8

Kobayashi, M., Hiyama, A., Miura, T., Asakawa, C., Hirose, M., \& Ifukube, T. (2011). Elderly user evaluation of mobile touchscreen interactions. In Human-Computer Interaction-INTERACT 2011 (pp. 83-99). Springer Berlin Heidelberg.

Llieva, J., Baron, S., \& Healey, N.M. (2002). Online surveys in marketing research: Pros and cons. International Journal of Market Research, 44, 361-367.

Makizako, H., Shimada, H., Park, H., Doi, T., Yoshida, D., Uemura, K., Tsutsumimoto, K., Suzuki, T. (2013). Evaluation of multidimensional neurocognitive function using a tablet personal computer: Test-retest reliability and validity in community-dwelling older adults. Geriatrics \& Gerontology International, 13, 860-866. doi: 10.1111/ggi.12014 
Minsker, S. (2014). The Cyclic Towers of Antwerpen problem: A challenging Hanoi variant. Discrete Applied Mathematics, 179, 44-53. doi: 10.1016/j.dam.2014.03.011

Musch, J., \& Reips, U.D. (2000). A brief history of Web experimenting. In M.H. Birnbaum (Ed). Psychological Experiments on the Internet (pp. 61-85). San Diego, CA; Academic Press.

Nelson, R.E., Dickson, A.L., \& Baños, J.H. (2000). An automated administration of Corsi's Block-Tapping test. Perceptual and Motor Skills, 91, 578-580.

Noyes, J.M., \& Garland, K.J. (2003). Solving the Tower of Hanoi: Does mode of presentation matter? Computers in Human Behavior, 19, 579-592. doi: 10.1016/S0747$5632(03) 00002-5$

Noyes, J.M., Garland, K.J., \& Robbins, E.L. (2004). Paper based versus computer based assessment: Is workload another test mode effect? British Journal of Educational Technology, 35, 111-113. doi: 10.1111/j.1467-8535.2004.00373.x

Phillips, L.H., Bull, R., Adams, E., \& Fraser, L. (2002). Positive mood and executive function: Evidence from stroop and fluency tasks. Emotion, 2, 12-22.

Piccardi, L., Iaria, G., Ricci, M., Bianchini, F., Zompanti, L., \& Guariglia, C. (2008). Walking in the Corsi test: Which type of memory do you need. Neuroscience Letters, 432, 127-131. doi: 10.1016/j.neulet.2007.12.044

Pruet, P., Ang, C.S., \& Farzin, D. (2015). Understanding tablet computer usage among primary school students in underdeveloped areas: Students' technology experience, learning styles and attitudes. Computers in Human Behavior, In Press. doi: 10.1016/j.chb.2014.09.063

Shackman, A.J., Sarinopoulos, I., Maxwell, J.S., Pizzagalli, D.A., Lavric, A., \& Davidson, R.J. (2006). Anxiety selectively disrupts visuospatial working memory. Emotion, 6, 40-61. doi: 10.1037/1528-3542.6.1.40 
Shah, D.S., Prados, J., Gamble, J., De Lillo, C., \& Gibson, CL. (2013). Sex differences in spatial memory using serial and search tasks. Behavioural Brain Research, 257, 9099. doi: 10.1016/j.bbr.2013.09.027

Shallice, T. (1982). Specific impairments of planning. Philosophical Transactions of the Royal Society, Series B, 298, 199-209. doi: 10.1098/rstb.1982.0082

Shneiderman, B. (1991). A taxonomy and rule base for the selection of interaction styles. Human factors for informatics usability, 325-342.

Siegenthaler, E., Bochud, Y., Wurtz, P., Schmid, L., \& Bergamin, P. (2012). The effects of touch screen technology on the usability of e-reading devices. Journal of Usability Studies, 7, 94-104.

Stanton, J.M. (2006). An empirical assessment of data collection using the Internet. Personnel Psychology, 51, 709-725. doi: 10.1111/j.1744-6570.1998.tb00259.x

Stoffers, D., Berendse, H.W., Deijen, J.B., \& Wolters, E., C.H. (2003). Deficits on Corsi's Block tapping task in early stage Parkinson's disease. Parkinsonism \& Related Disorders, 10, 107-111. doi: 10.1016/S1353-8020(03)00106-8

Straker, L.M., Coleman, J., Skoss, R., Maslen, B.A., Burgess-Limerick, R., \& Pollock, C.M. (2008). A comparison of posture and muscle activity during tablet computer, desktop computer and paper use by young children. Ergonomics, 51, 540-555. doi: $10.1080 / 00140130701711000$

Sung, E., \& Mayer, R.E. (2012). Students' beliefs about mobile devices Vs. desktop computers in South Korea and the United States. Computers \& Education, 59, 3281338.

Toy, K. J., Peres, S. C., David, T. Y., Nery, A., \& Phillips, R. G. (2012, September). Examining user preferences in interacting with touchscreen devices. In Proceedings 
of the Human Factors and Ergonomics Society Annual Meeting (Vol. 56, No. 1, pp. 1862-1866). SAGE Publications.

Vakil, E., Hassin-Baer, S., \& Karni, A. (2014). A deficit in optimizing task solution but robust and well-retained speed and accuracy gains in complex skill acquisition in Parkinson's disease: Multi-session training on the Tower of Hanoi Puzzle. Neuropsychologia, 57, 12-19. doi: 10.1016/j.neuropsychologia.2014.02.005 van der Linden, D., Frese, M., \& Meijman, T.F. (2003). Mental fatigue and the control of cognitive processes: effects on perseveration and planning. Acta Psychologica, 113, 45-65. doi: 10.1016/S0001-6918(02)00150-6

Vandierendonck, A., Kemps, E., Fastame, M.C., \& Szmalec, A. (2004). Working memory components of the Corsi blocks task. British Journal of Psychology, 95, 57-79. doi: $10.1348 / 000712604322779460$

Vasconcelos, A., Silva, P.A., Caseiro, J., Nunes, F., \& Teixeira, L.F. (2012). Designing tablet-based games for seniors: The example of CogniPlay, a cognitive gaming platform. Presented at International Conference on Fun and Games, Toulouse, September, 2012.

Wästlund, E., Reinikka, H., Norlander, T., \& Archer, T. (2005). Effects of VDT and paper presentation on consumption and production of information: Psychological and physiological factors. Computers in Human Behavior, 21, 377-394.

Welsh, M.C., \& Huizinga, M. (2005). Tower of Hanoi disk-transfer task: Influences of strategy knowledge and learning on performance. Learning and Individual Differences, 15, 283-298. doi: 10.1016/j.lindif.2005.05.002

Welsh, M. C., Satterlee-Cartmell, T., \& Stine, M. (1999). Towers of Hanoi and London: Contribution of working memory and inhibition to performance. Brain and Cognition, 41, 231-242. doi: 10.1006/brcg.1999.1123 
Williams, D. J., \& Noyes, J. M. (2007). Effect of experience and mode of presentation on problem solving. Computers in Human Behavior, 23, 258-274.

Wright, K.B. (2002). Social support within an online cancer community: An assessment of emotional support, perceptions of advantages and disadvantages, and motives for using the community. Journal of Applied Communication Research, 30, 195-209. doi: $10.1080 / 00909880216586$

Wright, L., \& Hardie, S.M. (2012). Are left-handers really more anxious? Laterality: Asymmetries of Body, Brain and Cognition, 17, 629-642. doi: 10.1080/1357650X.2011.615126

Yun, G.W., \& Trumbo, C.W. (2000). Comparative response to a survey executed by post, email, and web form. Journal of Computer Mediated Communication, 6. doi: 10.1111/j.1083-6101.2000.tb00112.x

Zickuhr, K. (2013). Tablet ownership 2013. Tablet, 19. Retrieved from http://www.pewinternet.org/files/oldmedia/Files/Reports/2013/PIP_Tablet\%20ownership\%202013.pdf 